\title{
HEAT EQUATION FROM MICROSCOPIC DYNAMICS: A WEAK COUPLING APPROACH
}

\author{
C. LIVERANI \\ Dipartimento di Mathematica, II Università di Roma, \\ Roma, 00133, Italy \\ E-mail: liverani@mat.uniroma2.it \\ www.university_name.edu \\ S. OLLA \\ CEREMADE, UMR CNRS 7534 \\ Université Paris Dauphine \\ Paris Cedex, 75775 France \\ E-mail: olla@ceremade.dauphine.fr
}

\begin{abstract}
We consider the dynamics of weakly coupled anharmonic oscillators, perturbed by a energy conserving noise. The noise considered exchange independently the components of the momentum of each oscillator. We prove that in the weak-coupling/long time limit energies of oscillators evolve following stochastic autonomous system of equations. Under a further diffusive space-time scaling of this system, the energy evolves following a nonlinear heat equation.
\end{abstract}

Keywords: Style file; LATEX; Proceedings; World Scientific Publishing.

\section{The quest for Heat equation, from the microscopic dynamics}

The mathematical rigorous deduction, from microscopic molecular dynamics, of heat equation, or of its stationary version called Fourier's law, is one of the main open problem in non equilibrium statistical mechanics [1].

To state the problem we can consider a chain of coupled anharmonic oscillators. This is described by the Hamiltonian

$$
\begin{aligned}
\mathcal{H}^{\Lambda} & =\sum_{i \in \Lambda}\left[\frac{\left|p_{i}\right|^{2}}{2}+\frac{1}{2} \sum_{j:|i-j|=1} V\left(q_{i}-q_{j}\right)+U\left(q_{i}\right)\right] \\
& =\sum_{i \in \Lambda} e_{i}
\end{aligned}
$$

where $\Lambda \subset \mathbb{Z}^{d}, q_{i}$ is the displacement of the oscillator $i$ from its equilibrium position, and $p_{i}$ its momentum (we fix masses equal to 1 ), $U$ is the anharmonic potential, and $V$ is the coupling potential. The Hamiltonian deterministic dynamics is as usual 
defined by the solution of

$$
\dot{q}_{i}=p_{i}, \quad \dot{p}_{i}=-\partial_{q_{i}} \mathcal{H}^{\Lambda}
$$

Then the mathematical problem can be stated, in a bit imprecise way, for the macroscopic evolution of the energy in a diffusive space-time scale (for simplicity of notation we consider here the case $d=1$ ):

Find a class of initial conditions such that, as $|\Lambda|=N \rightarrow \infty$, we have, for any continuous test function $G$ with compact support,

$$
\lim _{N \rightarrow \infty} \frac{1}{N} \sum_{i} G(i / N) e_{i}\left(N^{2} t\right)=\int G(y) T(y, t) d y,
$$

where $T(y, t)$ is the solution of the nonlinear heat equation

$$
\partial_{t} T=\nabla \kappa(T) \nabla T
$$

The thermal conductivity $\kappa(T)$ is defined by the Green-Kubo formula, i.e. can be obtained by the limit

$$
\kappa(T)=\lim _{t \rightarrow \infty} \frac{1}{2 T^{2} t} \sum_{i} i^{2}\left(<e_{i}(t) e_{0}(0)>_{T}-\bar{e}^{2}\right)
$$

where $<\cdot>_{T}$ stands here for the expectation of the infinite system in equilibrium at temperature $T$ (i.e. starting with the corresponding Gibbs measure). By using space and time translation invariance of $\left\langle\cdot>_{T}\right.$, thermal conductivity can be rewritten as

$$
\kappa(T)=\frac{1}{2 T^{2}} \int_{0}^{\infty} \sum_{i}\left\langle j_{i, i+1}(t) j_{0,1}(0)\right\rangle_{\beta} d t
$$

Here $j_{i, i+1}$ are the energy currents defined by

$$
\dot{e}_{i}(t)=\left(j_{i-1, i}(t)-j_{i, i+1}(t)\right)
$$

and explicitly given by

$$
j_{i, i+1}=-\frac{1}{2}\left(p_{i+1}+p_{i}\right) \cdot \nabla V\left(q_{i+1}-q_{i}\right) .
$$

At the moment this problem is completely open, and it is not even clear what could be the class of initial conditions under which it holds. Even the definition of the thermal conductivity $\kappa(T)$ is a challenging problem, since it requires information on the decay of correlations for an (infinite) deterministic system.

\section{Energy Conserving Noise}

It is clear that adding some noise to the dynamics should help. The nature of the noise should be such that it conserves energy, is local, and should give enough mixing properties to the dynamics to guarantee the convergence of the integrals in (6). We choose the noise as random forces that conserve the kinetic energy of each atom, given by independent diffusions on the spheres $\left\|p_{i}\right\|^{2}=$ cost). We consider the case 
where oscillators have two degree of freedom. In order to define such diffusions, consider the vector fields

$$
X_{i}:=p_{i}^{1} \partial_{p_{i}^{2}}-p_{i}^{2} \partial_{p_{i}^{1}}=: J p_{i} \cdot \partial_{p_{i}},
$$

and the second order operator

$$
S=\sum_{i \in \Lambda} X_{i}^{2}
$$

The generator of the process we are interested in is then given by

$$
L_{\Lambda}=: A+\sigma^{2} S
$$

where $A=\left\{H^{\Lambda}, \cdot\right\}$, the usual Hamiltonian operator. Notice that the effect of this random perturbation does not change the energy currents between oscillators.

Even with such noise the above mathematical problem is open and very challenging. What is clear in this case is that initial conditions will matter little, and the theorem should be valid for a large class of initial conditions.

\section{The weak coupling limit}

One way to attach the problem is to perform first a weak-coupling/large times limit of the system. Multiplying the coupling interaction $V$ by a small parameter $\varepsilon$, we obtain energy current of size $\epsilon$, and looking at time of order $\varepsilon^{-2} t$ we have for the evolution of the energies:

$$
e_{i}\left(\varepsilon^{-2} t\right)-e_{i}(0)=\sum_{|k-i|=1} \varepsilon \int_{0}^{\varepsilon^{-2} t} j_{i, k}(s) d s
$$

So it is clear that it is involved a central limit theorem problem for these very degenerate Markov processes. Here is our result:

Theorem 3.1. In the limit $\varepsilon \rightarrow 0$, the process $\left\{e_{i}\left(\varepsilon^{-2} t\right)\right\}_{i \in \Lambda}$ converges, in law, to the stochastic process $\left\{\mathcal{E}_{i}\right\}_{i \in \Lambda}$ determined by the stochastic differential equations

$$
d \mathcal{E}_{i}=\sum_{|i-k|=1} \alpha\left(\mathcal{E}_{i}, \mathcal{E}_{k}\right) d t+\sum_{|i-k|=1} \gamma\left(\mathcal{E}_{i}, \mathcal{E}_{k}\right) d B_{\{i, k\}}
$$

where $B_{\{i, k\}}=-B_{\{k, i\}}$ are independent standard Brownian motions.

The coefficients $\alpha$ and $\gamma$ are related by

$$
\alpha\left(a_{1}, a_{2}\right)=-\alpha\left(a_{2}, a_{1}\right)=\Gamma(\mathbf{a})^{-1}\left(\partial_{a_{1}}-\partial_{a_{2}}\right)\left[\Gamma(\mathbf{a}) \gamma\left(a_{1}, a_{2}\right)^{2}\right],
$$

with

$$
\Gamma(\mathbf{a})=\prod_{j} Z\left(a_{j}\right)
$$

and $Z(a)$ is a explicit function depending of $U$. 
We have that the generator of this dynamics can be written as

$$
\widetilde{L}=\frac{1}{2 \Gamma(\mathbf{a})} \sum_{i, j:|i-j|=1}\left(\partial_{a_{i}}-\partial_{a_{j}}\right)\left[\Gamma(\mathbf{a}) \gamma\left(a_{i}, a_{j}\right)^{2}\left(\partial_{a_{i}}-\partial_{a_{j}}\right)\right]
$$

In order to understand how closer we are now to heat equation, consider the harmonic case ( $U$ and $V$ quadratic). In this case these coefficient can be explicitly computed

$$
\gamma\left(a_{i}, a_{j}\right)^{2}=a_{i} a_{j}, \quad \alpha\left(a_{i}, a_{j}\right)=-\left(a_{i}-a_{j}\right)
$$

and the corresponding stochastic differential equations became

$$
\begin{aligned}
d \mathcal{E}_{i}(t)= & \sum_{|i-j|=1}-\left(\mathcal{E}_{i}(t)-\mathcal{E}_{j}(t)\right) d t+\sum_{|i-j|=1} \sqrt{\mathcal{E}_{i}(t) \mathcal{E}_{j}(t)} d w_{i, j}(t) \\
& =\Delta \mathcal{E}_{i}(t) d t+\sum_{|i-j|=1} \sqrt{\mathcal{E}_{i}(t) \mathcal{E}_{j}(t)} d w_{i, j}(t)
\end{aligned}
$$

i.e. we have already heat equation in this time-scale, plus a diffusive term. In the non-linear case, $\alpha\left(a_{i}, a_{j}\right)$ is not a difference, and this dynamics falls in the class of conservative stochastic dynamics called non-gradient Ginszburg-Landau models.

\section{The hydrodynamic limit}

The process (10) is close the the one studied by Varadhan in [2]. In this paper Varadhan proves an hydrodynamic limit, i. e. that under certain condition on the initial distribution, for any test function $G$ on $\mathbb{R}$ we have the convergence

$$
\lim _{N \rightarrow \infty} \frac{1}{N} \sum_{i} G(i / N) \mathcal{E}_{i}\left(N^{2} t\right)=\int G(y) u(y, t) d y
$$

where $u(y, t)$ is the solution of a nonlinear heat equation

$$
\partial_{t} u=\nabla D(u) \nabla u
$$

Yet our case it is not covered by such result (due to the degeneracy at zero of the diffusion coefficients and the non strict convexity of the potential of the invariant measure). In any case the extension of Varadhan's work to the present case would allow to obtain the heat equation in the present setting via a diffusive limit. We plan to work on such an extension in the future.

\section{References}

[1] R.-B. F. Bonetto, J.L. Lebowitz, Fourier's law: A challenge to theorists, in Mathematical Physics 2000, (Imperial College Press, London,, 2000).

[2] S. Varadhan, Nonlinear diffusion limit for a system with nearest neighbor interactions. ii, in Asymptotic problems in probability theory: stochastic models and diffusions on fractals, (Sanda/Kyoto, 1990), ed. H. Longman Sci. Tech., Pitman Res. Notes Math. Ser., Vol. 2831993. 